\title{
Chemical Composition and Thickness Retrieval in HRTEM by a Reversed Multislice Process
}

\author{
Fu-Rong Chen ${ }^{1}$, Christian Kisielowski ${ }^{2}$, Joerg R. Jinschek ${ }^{2}$, J. Plitzko ${ }^{3}$, and Ji-Jung Kai ${ }^{1}$
}

1. Dept. of Engineering and System Science, National Tsing Hua University, Hsin-Chu, Taiwan

2. National Center for Electron Microscopy, LBNL, One Cyclotron Rd. Berkeley CA 94720, USA

3. Max-Planck-Institut für Biochemie, Am Klopferspitz 18, D-82152 Martinsried

In general terms, lattice images do not reveal the real atomic structure of the sample directly, can be distorted, and information about the sample thickness and chemical composition is heavily encoded. These drawbacks relate to the presence of lens aberrations and dynamic diffraction. The development of Cs corrected TEM [1,2] and software that reconstructs the complex electron exit wave function [3-7] aim at removing lens aberrations. Several approaches, such as the 1s state model [8], reversing multislice algorithms [9,10], or simulated annealing and maximum likelihood algorithms [11, 12], have been proposed to remove the dynamical scattering effect and to retrieve the crystal potential from the complex exit wave. In this paper, we present a new method to retrieve the potential map from the exit wave based on reversing multi-slice calculations. This algorithm uses a non-linear optimization scheme to find an optimum phase grating that satisfies two boundary conditions: knowledge of the entrance surface wave and the measured exit surface wave. The exit wave of a wedge shaped Au crystal and an $\mathrm{Al}(10 \% \mathrm{Cu})$ crystal were simulated to test this algorithm. Good agreement between the recovered crystal potentials and input parameters was found up to a thickness where phase reversal occurs because of dynamic scattering. After the phase grating is retrieved, the position of atom columns and their chemical composition can be quantified. Compositional maps $X_{a}(r)$ and $X_{b}(r)$ of binary alloys can be deduced from the crystal potential map $\mathrm{V}(\mathrm{r})$ using the linear relation $\mathrm{V}(\mathrm{r})=\mathrm{X}_{\mathrm{a}}(\mathrm{r}) \mathrm{V}_{\mathrm{a}}+\mathrm{X}_{\mathrm{b}}(\mathrm{r}) \mathrm{V}_{\mathrm{b}}$, where $\mathrm{V}_{\mathrm{a}}$ and $\mathrm{V}_{\mathrm{b}}$ are the mean inner potentials of the element $\mathrm{A}$ and $\mathrm{B}$, respectively. Figure 1 (a) and (b) show the phase of an electron exit wave of an $\mathrm{Al}: \mathrm{Cu}$ bi-crystal and an $\mathrm{InGaN} / \mathrm{GaN}$ quantum well that were reconstructed from focal series of 20 images each. Figure 2 (a) and (b) shows the retrieved potential maps of the $\mathrm{Al}$ and $\mathrm{Cu}$ atoms, respectively, and sitespecific $\mathrm{Cu}$ segregation to the boundary is revealed. Fig. 3 (a) and (b) depicts Ga and In maps from the quantum well region. It is clear from both cases that chemical differences can be distinguished on a single atom level due to the different atomic number $\mathrm{Z}$ of the elements. Limitations of the algorithm due to systematic and statistical errors will be discussed.

\section{References:}

[1] H. Rose, Optik 85 (1) (1990) 19.

[2] M. Haider, H. Rose, S. Uhlemann, E. Schwan, B. Kabius, K. Urban, Ultramicroscopy 23 (1998) 768.

[3] W.O. Saxton, in: Advances in Electronics and Electron Physics, Computer Techniques for Image Processing in Electron Microcopy, Academic Press, New York, 1978.

[4] E.J. Kirkland, B.M. Siegel, N. Uyeda, Y. Fujiyoshi, Ultramicroscopy 17 (1985) 87.

[5] M. Op De Beeck, D. Van Dyck, W. Coene, Ultramicroscopy 64 (1996) 167.

[6] W.-K. Hsieh, Fu-Rong Chen, Ji-Jung Kai and A. I. Kirkland, Ultramicroscopy 98 (2004) 99

[7] Les Allen et al, Ultramicroscopy 100 (2004) 91-104

[8] M. Op De Beeck and D. Van Dyck, Phys. Stat. Sol., 150 (1995) 587

[9] M. J. Beeching and A. E. C. Spargo, Ultramicroscopy 52 (1993) 243

[10] A. E. C. Spargo, M. J. Beeching and L. J. Allen, Ultramicroscopy 55 (1994) 329

[11] M. Lentzen and K. Urban, Ultramicroscopy, 62 (1996) 89-102

[12] M. Lentzen and K. Urban, Acta Crystallography, A56, (2000), 235-247 

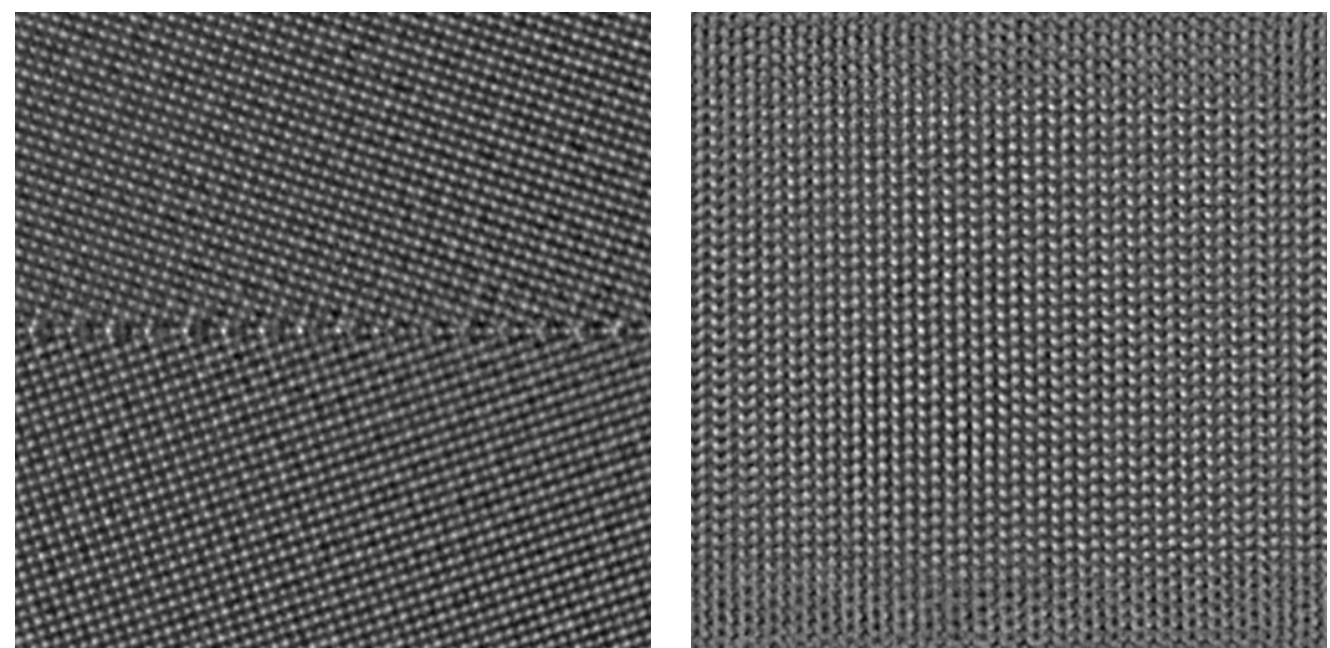

Fig. 1 The phase of the exit wave of grain boundary in (a) $\mathrm{Al}(\mathrm{Cu})$ [100] (b) InGaN/GaN Quantum well

QuickTime ${ }^{T M}$ and a
TIFF (LZW) decompressor
are needed to see this picture.

Fig. 2 Retrieved compositional maps: (a) Al (left), (b) $\mathrm{Cu}$ (middle). The segregation of $\mathrm{Cu}$ to the grain boundary can be quantified by an averaged composition profiles (the detail will be shown in the talk)

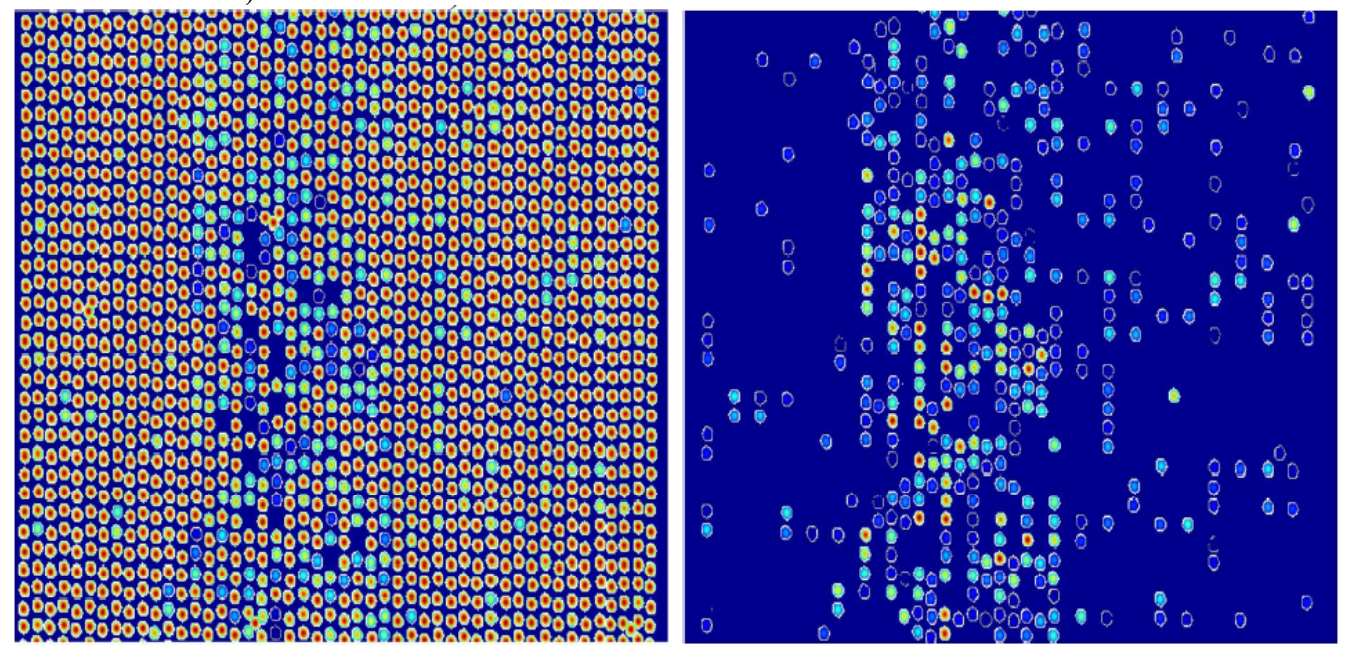

Fig.3. Retrieved compositional maps: (a) Ga (left), (b) In (middle). (the detail will be shown in the talk. 